\title{
On the oscillation of $q$-fractional difference equations
}

Bahaaeldin Abdalla*

\author{
"Correspondence: \\ babdallah@psu.edu.sa \\ Department of Mathematics and \\ Physical Sciences, Prince Sultan \\ University, P. O. Box 66833, Riyadh, \\ 11586, Saudi Arabia
}

\begin{abstract}
In this paper, sufficient conditions are established for the oscillation of solutions of $q$-fractional difference equations of the form

$$
\left\{\begin{array}{l}
{ }_{q} \nabla_{0}^{\alpha} x(t)+f_{1}(t, x)=r(t)+f_{2}(t, x), \quad t>0, \\
\lim _{t \rightarrow 0^{+}} q^{j-\alpha} x(t)=b_{j} \quad(j=1,2, \ldots, m),
\end{array}\right.
$$

where $m=\lceil\alpha\rceil,{ }_{q} \nabla_{0}^{\alpha}$ is the Riemann-Liouville $q$-differential operator and $l_{0}^{m-\alpha}$ is the $q$-fractional integral. The results are also obtained when the Riemann-Liouville $q$-differential operator is replaced by Caputo $q$-fractional difference. Examples are provided to demonstrate the effectiveness of the main result.
\end{abstract}

MSC: 39A13; 39A21; 26A33

Keywords: $q$-fractional difference equations; oscillation theory

\section{Introduction}

The oscillation theory for fractional differential equations was initiated in [1] where oscillation criteria were obtained for a nonlinear fractional differential equation of the form

$$
\left\{\begin{array}{l}
D_{a}^{\alpha} x(t)+f_{1}(t, x)=v(t)+f_{2}(t, x), \quad t>a, \\
D_{a}^{\alpha-k} x(a)=b_{k} \quad(k=1,2, \ldots, m-1), \quad \lim _{t \rightarrow a^{+}} I_{a}^{m-\alpha} x(t)=b_{m}
\end{array}\right.
$$

where $m=\lceil\alpha\rceil, m \in \mathbb{N}, D_{a}^{\alpha}$ is the Riemann-Liouville fractional derivative of order $\alpha$ and $I_{a}^{m-\alpha}$ is the Riemann-Liouville fractional integral of order $m-\alpha$ of $x$. The oscillation of (1) was extended in [2], and more general cases were studied. On the other hand, the oscillation of solutions for fractional difference equations, which is the discrete counterpart of the corresponding fractional differential equations, was first studied in [3]. Indeed, sufficient conditions were given for the oscillation of solutions for fractional difference equations of the form

$$
\left\{\begin{array}{l}
\nabla_{a(\alpha)-1}^{\alpha} x(t)+f_{1}(t, x)=v(t)+f_{2}(t, x), \quad t \in \mathbb{N}_{a(\alpha)}, \\
\left.\nabla_{a(\alpha)-1}^{-(m-\alpha)} x(t)\right|_{t=a(\alpha)}=x(a(\alpha))=c, \quad c \in \mathbb{R}
\end{array}\right.
$$

where $m=\lceil\alpha\rceil, m \in \mathbb{N}, \nabla_{a(\alpha)}^{\alpha}$ is the Riemann-Liouville difference operator of order $\alpha$ and $\nabla_{a(\alpha)}^{-\alpha}$ is the Riemann-Liouville sum operator. The oscillation of (2) was extended in [4]. The

(c) The Author(s) 2017. This article is distributed under the terms of the Creative Commons Attribution 4.0 International License (http://creativecommons.org/licenses/by/4.0/), which permits unrestricted use, distribution, and reproduction in any medium, provided you give appropriate credit to the original author(s) and the source, provide a link to the Creative Commons license, and indicate if changes were made. 
extensive investigations amongst differential and difference equations intuitively lead to developments in the direction of $q$-fractional calculus which deals with fractional derivatives and integrals with arbitrary order on the quantum time scale. It has been proved that the theory of $q$-calculus has lots of applications in many fields of science and engineering. For some of these applications, we refer to [5-12].

Following this trend and motivated by the claim that there are no results available in the literature regarding the oscillation of solutions of $q$-fractional difference equations, we consider equations of the form

$$
\left\{\begin{array}{l}
{ }_{q} \nabla_{0}^{\alpha} x(t)+f_{1}(t, x)=r(t)+f_{2}(t, x), \quad t>0, \\
\lim _{t \rightarrow 0^{+}} q_{0}^{j-\alpha} x(t)=b_{j} \quad(j=1,2, \ldots, m),
\end{array}\right.
$$

where $m=\lceil\alpha\rceil,{ }_{q} \nabla_{0}^{\alpha}$ and ${ }_{q} I_{0}^{m-\alpha}$ denote the Riemann-Liouville $q$-differential and integral operators which will be defined later.

The objective of this paper is to carry out the results obtained in the above mentioned papers to the $q$-fractional difference equations of the form (3). This paper is organized as follows. Section 2 introduces some notations and provides the definitions of the $q$ fractional integral and differential operators together with some basic properties and lemmas that are needed in the proofs of the main theorems. In Section 3, the main theorems are presented. Section 4 is devoted to the results obtained when the Riemann-Liouville $q$-differential operator is replaced by Caputo $q$-fractional difference. Two examples are provided in Section 5 to demonstrate the effectiveness of the main theorems.

\section{Notations and preliminary assertions}

For $0<q<1$, let $T_{q}$ be the time scale

$$
T_{q}=\left\{q^{n}: n \in \mathbb{Z}\right\} \cup\{0\} \subset[0, \infty) .
$$

For a function $f: T_{q} \rightarrow \mathbb{R}$, the nabla $q$-derivative of $f$ is given by

$$
\nabla_{q} f(t)=\frac{f(t)-f(q t)}{(1-q) t}, \quad t \in T_{q}-\{0\}
$$

The nabla $q$-integral of $f$ is given by

$$
\int_{0}^{t} f(s) \nabla_{q} s=(1-q) t \sum_{i=0}^{\infty} q^{i} f\left(t q^{i}\right) .
$$

From the theories of $q$-calculus and time scale, the following product rule is valid:

$$
\nabla_{q}(f(t) g(t))=f(q t) \nabla_{q} g(t)+\left(\nabla_{q} f(t)\right) g(t)
$$

The $q$-analogue of the power function $(t-s)^{n}$ with $n \in \mathbb{N}$ is

$$
(t-s)_{q}^{0}=1, \quad(t-s)_{q}^{n}=\prod_{i=0}^{n-1}\left(t-q^{i} s\right) .
$$


More generally, when $\alpha$ is any real number, then

$$
(t-s)_{q}^{\alpha}=t^{\alpha} \prod_{i=0}^{\infty} \frac{1-\frac{s}{t} q^{i}}{1-\frac{s}{t} q^{i+\alpha}} .
$$

Note that if $t \leq s$, then $(t-s)_{q}^{\alpha}=0$ and if $s=0$, then $t_{q}^{\alpha}=t^{\alpha}$.

Lemma 2.1 (see [13]) For $\alpha, \gamma, \beta \in \mathbb{R}$, one has the following.

1. $(t-s)_{q}^{\beta+\gamma}=(t-s)_{q}^{\beta}\left(t-q^{\beta} s\right)_{q}^{\gamma}$;

2. $(a t-a s)_{q}^{\beta}=a^{\beta}(t-s)_{q}^{\beta}$;

3. the nabla q-derivative of the q-factorial function with respect to $t$ is

$$
\nabla_{q}(t-s)_{q}^{\alpha}=\frac{1-q^{\alpha}}{1-q}(t-s)_{q}^{\alpha-1}
$$

4. the nabla q-derivative of the q-factorial function with respect to $s$ is

$$
\nabla_{q}(t-s)_{q}^{\alpha}=-\frac{1-q^{\alpha}}{1-q}(t-q s)_{q}^{\alpha-1}
$$

Moreover, the $q$-fractional integral of order $\alpha \neq 0,-1,-2, \ldots$ is defined by

$$
{ }_{q} I_{0}^{\alpha} f(t)=\frac{1}{\Gamma_{q}(\alpha)} \int_{0}^{t}(t-q s)_{q}^{\alpha-1} f(s) \nabla_{q} s
$$

where

$$
\Gamma_{q}(\alpha)=\frac{(1-q)_{q}^{\alpha-1}}{(1-q)^{\alpha-1}}, \quad \Gamma_{q}(\alpha+1)=\frac{1-q^{\alpha}}{1-q} \Gamma_{q}(\alpha) \quad \text { and } \quad \Gamma_{q}(1)=1 .
$$

Let $\alpha>0$. If $\alpha \notin \mathbb{N}$, then the $\alpha$-order Liouville-Caputo (left) $q$-fractional derivative of a function $f$ is defined by (see [6])

$$
{ }_{q} C_{a}^{\alpha} f(t) \triangleq{ }_{q} I_{a}^{(n-\alpha)} \nabla_{q}^{n} f(t)=\frac{1}{\Gamma(n-\alpha)} \int_{a}^{t}(t-q s)_{q}^{n-\alpha-1} \nabla_{q}^{n} f(s) \nabla_{q} s
$$

where $n=\lceil\alpha\rceil$ and $\lceil\alpha\rceil$ denotes the smallest integer greater than or equal to $\alpha$. If $\alpha \in \mathbb{N}$, then ${ }_{q} C_{a}^{\alpha} f(t) \triangleq \nabla_{q}^{n} f(t)$.

Let $\alpha>0$. If $\alpha \notin \mathbb{N}$, then the $\alpha$-order Riemann (left) $q$-fractional derivative of a function $f$ is defined by

$$
{ }_{q} \nabla_{a}^{\alpha} f(t) \triangleq \nabla_{q}^{n} I_{a}^{(n-\alpha)} f(t)
$$

The following identity is useful to transform Caputo $q$-fractional difference equation into nabla $q$-fractional sum.

Let $\alpha>0$ and $f$ be defined in suitable domains. Then (see [6])

$$
{ }_{q} I_{a}^{\alpha} C_{a}^{\alpha} f(t)=f(t)-\sum_{k=0}^{n-1} \frac{(t-a)_{q}^{k}}{\Gamma_{q}(k+1)} \nabla_{q}^{k} f(a),
$$


and if $0<\alpha \leq 1$ then

$$
{ }_{q} I_{a q}^{\alpha} C_{a}^{\alpha} f(t)=f(t)-f(a)
$$

Moreover, it is clear that ${ }_{q} I_{a q}^{\alpha} \nabla_{a}^{\alpha} f(t)=f(t)$. The following identity is essential to solving linear $q$-fractional equations:

$$
{ }_{q} I_{a}^{\alpha}(x-a)_{q}^{\mu}=\frac{\Gamma_{q}(\mu+1)}{\Gamma_{q}(\alpha+\mu+1)}(x-a)_{q}^{\mu+\alpha} \quad(0 \leq a<x<b),
$$

where $\alpha \in \mathbb{R}^{+}$and $\mu \in(-1, \infty)$.

Lemma 2.2 ([14] (Young's inequality))

(i) Let $X, Y \geq 0, u>1$ and $\frac{1}{u}+\frac{1}{v}=1$, then $X Y \leq \frac{1}{u} X^{u}+\frac{1}{v} Y^{v}$.

(ii) Let $X \geq 0, Y>0,0<u<1$ and $\frac{1}{u}+\frac{1}{v}=1$, then $X Y \geq \frac{1}{u} X^{u}+\frac{1}{v} Y^{v}$, where equalities hold if and only if $Y=X^{u-1}$.

\section{Oscillation of Riemann $q$-fractional difference equations}

In this section we study the oscillation theory for equation (3). Let $\mathcal{L}_{q}^{1}[0, b]$ denote the space of all functions $f$ defined on $(0, b]$ satisfying $\|f\|:=\sup _{x \in(0, b]} \int_{0}^{x}|f(t)| d_{q} t<\infty$, and let $\mathcal{A} C_{q}^{(n)}[0, b]$ denote the space of all functions $f$ defined on $[0, b]$ such that $f, D_{q} f, \ldots, D_{q}^{n-1} f$ are $q$-regular at zero and $D_{q}^{n-1} f(x) \in \mathcal{A} C_{q}^{(1)}[0, b]$.

Lemma 3.1 ([5]) Let $\alpha \in \mathbb{R}^{+}$and $n:=\lceil\alpha\rceil$.Iff $\in \mathcal{L}_{q}^{1}[0, b]$ such that $I_{q}^{n-\alpha} f \in \mathcal{A} C_{q}^{(n)}[0, b]$, then

$$
I_{q}^{\alpha} D_{q}^{\alpha} f(x)=f(x)-\sum_{j=1}^{n} D_{q}^{\alpha-j} f\left(0^{+}\right) \frac{x^{\alpha-j}}{\Gamma_{q}(\alpha-j+1)}, \quad x \in(0, b] .
$$

Using Lemma 3.1, the solution representation of (3) can be written as

$$
x(t)=\sum_{j=1}^{m} b_{j} \frac{t^{\alpha-j}}{\Gamma_{q}(\alpha-j+1)}+{ }_{q} I_{0}^{\alpha} F(t, x),
$$

where $F(t, x)=r(t)+f_{2}(t, x)-f_{1}(t, x)$.

A solution of (3) is said to be oscillatory if it has arbitrarily large zeros on $(0, \infty)$; otherwise, it is called nonoscillatory. An equation is said to be oscillatory if all of its solutions are oscillatory.

We prove our results under the following assumptions:

$$
\begin{aligned}
& x f_{i}(t, x)>0 \quad(i=1,2), x \neq 0, t \geq 0, \\
& \left|f_{1}(t, x)\right| \geq p_{1}(t)|x|^{\beta} \quad \text { and } \quad\left|f_{2}(t, x)\right| \leq p_{2}(t)|x|^{\gamma}, \quad x \neq 0, t \geq 0, \\
& \left|f_{1}(t, x)\right| \leq p_{1}(t)|x|^{\beta} \quad \text { and } \quad\left|f_{2}(t, x)\right| \geq p_{2}(t)|x|^{\gamma}, \quad x \neq 0, t \geq 0,
\end{aligned}
$$

where $p_{1}, p_{2} \in C([0, \infty),(0, \infty))$ and $\beta, \gamma$ are positive constants.

Define

$$
\Phi(t)=\Gamma_{q}(\alpha) \sum_{j=1}^{m} b_{j} \frac{t^{\alpha-j}}{\Gamma_{q}(\alpha-j+1)}
$$


and

$$
\Psi\left(t, T_{1}\right)=\int_{0}^{T_{1}}(t-q s)_{q}^{\alpha-1} F(s, x(s)) \nabla_{q} s
$$

Theorem 3.2 Let $f_{2}=0$ in (3) and condition (13) hold. If

$$
\liminf _{t \rightarrow \infty} t^{1-\alpha} \int_{0}^{t}(t-q s)_{q}^{\alpha-1} r(s) \nabla_{q} s=-\infty
$$

and

$$
\limsup _{t \rightarrow \infty} t^{1-\alpha} \int_{0}^{t}(t-q s)_{q}^{\alpha-1} r(s) \nabla_{q} s=\infty
$$

then every solution of $(3)$ is oscillatory.

Proof Let $x(t)$ be a nonoscillatory solution of equation (3) with $f_{2}=0$. Suppose that $T_{1}>0$ is large enough so that $x(t)>0$ for $t \geq T_{1}$. Hence, (13) implies that $f_{1}(t, x)>0$ for $t \geq T_{1}$. Using (7), we get from (12)

$$
\begin{aligned}
\Gamma_{q}(\alpha) x(t)= & \Gamma_{q}(\alpha) \sum_{j=1}^{m} b_{j} \frac{t^{\alpha-j}}{\Gamma_{q}(\alpha-j+1)}+\int_{0}^{T_{1}}(t-q s)_{q}^{\alpha-1} F(s, x(s)) \nabla_{q} s \\
& +\int_{T_{1}}^{t}(t-q s)_{q}^{\alpha-1}\left[r(s)-f_{1}(s, x(s))\right] \nabla_{q} s \\
\leq & \Phi(t)+\Psi\left(t, T_{1}\right)+\int_{T_{1}}^{t}(t-q s)_{q}^{\alpha-1} r(s) \nabla_{q} s
\end{aligned}
$$

where $\Phi$ and $\Psi$ are defined in (16) and (17), respectively. Multiplying (20) by $t^{1-\alpha}$, we get

$$
0<t^{1-\alpha} \Gamma_{q}(\alpha) x(t) \leq t^{1-\alpha} \Phi(t)+t^{1-\alpha} \Psi\left(t, T_{1}\right)+t^{1-\alpha} \int_{T_{1}}^{t}(t-q s)_{q}^{\alpha-1} r(s) \nabla_{q} s
$$

Take $T_{2}>T_{1}$. We consider two cases.

Case (1): Let $0<\alpha \leq 1$. Then $m=1, \Phi(t)=b_{1} t^{\alpha-1}$ hence

$$
t^{1-\alpha} \Phi(t)=\left|b_{1}\right|
$$

The product rule (4) and Lemma 2.1 can be used to show that the function $h(t)=$ $t^{1-\alpha}(t-q s)_{q}^{\alpha-1}$ is decreasing for $\alpha \in(0,1)$. Then

$$
\begin{aligned}
\left|t^{1-\alpha} \Psi\left(t, T_{1}\right)\right| & =\left|t^{1-\alpha} \int_{0}^{T_{1}}(t-q s)_{q}^{\alpha-1}\left[r(s)+f_{2}(s, x(s))-f_{1}(s, x(s))\right] \nabla_{q} s\right| \\
& \leq \int_{0}^{T_{1}} t^{1-\alpha}(t-q s)_{q}^{\alpha-1}\left|r(s)+f_{2}(s, x(s))-f_{1}(s, x(s))\right| \nabla_{q} s \\
& \leq \int_{0}^{T_{1}} T_{2}^{1-\alpha}\left(T_{2}-q s\right)_{q}^{\alpha-1}\left|r(s)+f_{2}(s, x(s))-f_{1}(s, x(s))\right| \nabla_{q} s \\
& :=c_{1}\left(T_{1}, T_{2}\right) \quad \text { for } t \geq T_{2} .
\end{aligned}
$$


Then, from equation (21) and for $t \geq T_{2}$, we get

$$
t^{1-\alpha} \int_{T_{1}}^{t}(t-q s)_{q}^{\alpha-1} r(s) \nabla_{q} s \geq-\left[\left|b_{1}\right|+c_{1}\left(T_{1}, T_{2}\right)\right]
$$

hence

$$
\liminf _{t \rightarrow \infty} t^{1-\alpha} \int_{T_{1}}^{t}(t-q s)_{q}^{\alpha-1} r(s) \nabla_{q} s \geq-\left[\left|b_{1}\right|+c_{1}\left(T_{1}, T_{2}\right)\right]>-\infty
$$

which contradicts condition (18).

Case (2): Let $\alpha>1$. Then $m \geq 2$, hence for $t \geq T_{2}$ we have

$$
\begin{aligned}
\left|t^{1-\alpha} \Phi(t)\right| & =\left|t^{1-\alpha} \Gamma_{q}(\alpha) \sum_{j=1}^{m} b_{j} \frac{t^{\alpha-j}}{\Gamma_{q}(\alpha-j+1)}\right| \\
& \leq \Gamma_{q}(\alpha) \sum_{j=1}^{m}\left|b_{j}\right| \frac{t^{1-j}}{\Gamma_{q}(\alpha-j+1)} \\
& \leq \Gamma_{q}(\alpha) \sum_{j=1}^{m}\left|b_{j}\right| \frac{T_{2}^{1-j}}{\Gamma_{q}(\alpha-j+1)}:=c_{2}\left(T_{2}\right) .
\end{aligned}
$$

Using (6) with $\alpha>1$, we easily note that $t^{1-\alpha}(t-q s)_{q}^{\alpha-1}<1$, hence, for $t \geq T_{2}$, we have

$$
\begin{aligned}
\left|t^{1-\alpha} \Psi\left(t, T_{1}\right)\right| & =\left|t^{1-\alpha} \int_{0}^{T_{1}}(t-q s)_{q}^{\alpha-1}\left[r(s)+f_{2}(s, x(s))-f_{1}(s, x(s))\right] \nabla_{q} s\right| \\
& \leq \int_{0}^{T_{1}} t^{1-\alpha}(t-q s)_{q}^{\alpha-1}\left|r(s)+f_{2}(s, x(s))-f_{1}(s, x(s))\right| \nabla_{q} s \\
& \leq \int_{0}^{T_{1}}\left|r(s)+f_{2}(s, x(s))-f_{1}(s, x(s))\right| \nabla_{q} s:=c_{3}\left(T_{1}\right) .
\end{aligned}
$$

From (21), (24) and (25), we conclude that

$$
t^{1-\alpha} \int_{T_{1}}^{t}(t-q s)_{q}^{\alpha-1} r(s) \nabla_{q} s \geq-\left[c_{2}\left(T_{2}\right)+c_{3}\left(T_{1}\right)\right]
$$

for $t \geq T_{2}$. Hence, we obtain

$$
\liminf _{t \rightarrow \infty} t^{1-\alpha} \int_{T_{1}}^{t}(t-q s)_{q}^{\alpha-1} r(s) \nabla_{q} s \geq-\left[c_{2}\left(T_{2}\right)+c_{3}\left(T_{1}\right)\right]>-\infty,
$$

which contradicts condition (18). Therefore, we conclude that $x(t)$ is oscillatory. In case $x(t)$ is eventually negative, similar arguments lead to a contradiction with condition (19).

Theorem 3.3 Let conditions (13) and (14) hold with $\beta>\gamma$. If

$$
\liminf _{t \rightarrow \infty} t^{1-\alpha} \int_{T}^{t}(t-q s)_{q}^{\alpha-1}[r(s)+H(s)] \nabla_{q} s=-\infty
$$


and

$$
\limsup _{t \rightarrow \infty} t^{1-\alpha} \int_{T}^{t}(t-q s)_{q}^{\alpha-1}[r(s)-H(s)] \nabla_{q} s=\infty
$$

for every sufficiently large $T$, where

$$
H(s)=\frac{\beta-\gamma}{\gamma} p_{1}^{\frac{\gamma}{\gamma-\beta}}(s)\left[\frac{\gamma p_{2}(s)}{\beta}\right]^{\frac{\beta}{\beta-\gamma}},
$$

then every solution of $(3)$ is oscillatory.

Proof Let $x(t)$ be a nonoscillatory solution of equation (3), say, $x(t)>0$ for $t \geq T_{1}>0$. Let $s \geq T_{1}$. Using conditions (13) and (14), we get $f_{2}(s, x)-f_{1}(s, x) \leq p_{2}(s) x^{\gamma}(s)-p_{1}(s) x^{\beta}(s)$. Let $X=x^{\gamma}(s), Y=\frac{\gamma p_{2}(s)}{\beta p_{1}(s)}, u=\frac{\beta}{\gamma}$ and $v=\frac{\beta}{\beta-\gamma}$, then from part (i) of Lemma 2.2 we get

$$
\begin{aligned}
p_{2}(s) x^{\gamma}(s)-p_{1}(s) x^{\beta}(s) & =\frac{\beta p_{1}(s)}{\gamma}\left[x^{\gamma}(s) \frac{\gamma p_{2}(s)}{\beta p_{1}(s)}-\frac{\gamma}{\beta}\left(x^{\gamma}(s)\right)^{\frac{\beta}{\gamma}}\right] \\
& =\frac{\beta p_{1}(s)}{\gamma}\left[X Y-\frac{1}{u} X^{u}\right] \leq \frac{\beta p_{1}(s)}{\gamma} \frac{1}{v} Y^{v}=H(s),
\end{aligned}
$$

where $H$ is defined as in Theorem (3.3). Then from equation (12) we obtain

$$
\begin{aligned}
\Gamma_{q}(\alpha) x(t) & =\Phi(t)+\Psi\left(t, T_{1}\right)+\int_{T_{1}}^{t}(t-q s)_{q}^{\alpha-1}\left[r(s)+f_{2}(s, x(s))-f_{1}(s, x(s))\right] \nabla_{q} s \\
& \leq \Phi(t)+\Psi\left(t, T_{1}\right)+\int_{T_{1}}^{t}(t-q s)_{q}^{\alpha-1}\left[r(s)+p_{2}(s) x^{\gamma}(s)-p_{1}(s) x^{\beta}(s)\right] \nabla_{q} s \\
& \leq \Phi(t)+\Psi\left(t, T_{1}\right)+\int_{T_{1}}^{t}(t-q s)_{q}^{\alpha-1}[r(s)+H(s)] \nabla_{q} s \quad \text { for } t \geq T_{1} .
\end{aligned}
$$

The rest of the proof is the same as that of Theorem 3.2 and hence is omitted.

Theorem 3.4 Let $\alpha \geq 1$ and suppose that (13) and (15) hold with $\beta<\gamma$. If

$$
\limsup _{t \rightarrow \infty} t^{1-\alpha} \int_{T}^{t}(t-q s)_{q}^{\alpha-1}[r(s)+H(s)] \nabla_{q} s=\infty
$$

and

$$
\liminf _{t \rightarrow \infty} t^{1-\alpha} \int_{T}^{t}(t-q s)_{q}^{\alpha-1}[r(s)-H(s)] \nabla_{q} s=-\infty
$$

for every sufficiently large $T$, where $H$ is defined as in Theorem 3.3, then every bounded solution of (3) is oscillatory.

Proof Let $x(t)$ be a bounded nonoscillatory solution of equation (3). Then there exist constants $M_{1}$ and $M_{2}$ such that

$$
M_{1} \leq x(t) \leq M_{2} \quad \text { for } t \geq 0
$$


Assume that $x$ is a bounded eventually positive solution of (3). Then there exists $T_{1}>0$ such that $x(t)>0$ for $t \geq T_{1}>0$. Using conditions (13) and (15), we get $f_{2}(s, x)-f_{1}(s, x) \geq$ $p_{2}(s) x^{\gamma}(s)-p_{1}(s) x^{\beta}(s)$. Using (ii) of Lemma 2.2 and similar to the proof of (28), we find

$$
p_{2}(s) x^{\gamma}(s)-p_{1}(s) x^{\beta}(s) \geq H(s) \quad \text { for } s \geq T_{1} .
$$

From (12) and similar to (29), we obtain

$$
\Gamma_{q}(\alpha) x(t) \geq \Phi(t)+\Psi\left(t, T_{1}\right)+\int_{T_{1}}^{t}(t-q s)_{q}^{\alpha-1}[r(s)+H(s)] \nabla_{q} s
$$

Multiplying by $t^{1-\alpha}$, we get

$$
t^{1-\alpha} \Gamma_{q}(\alpha) x(t) \geq t^{1-\alpha} \Phi(t)+t^{1-\alpha} \Psi\left(t, T_{1}\right)+t^{1-\alpha} \int_{T_{1}}^{t}(t-q s)_{q}^{\alpha-1}[r(s)+H(s)] \nabla_{q} s .
$$

Take $T_{2}>T_{1}$. We consider two cases.

Case (1): Let $\alpha=1$. Then (22) and (23) are still correct. Hence, from (33) and using (32), we find that

$$
M_{2} \Gamma_{q}(\alpha) \geq t^{1-\alpha} \Gamma_{q}(\alpha) x(t) \geq-\left|b_{1}\right|-c_{1}\left(T_{1}, T_{2}\right)+t^{1-\alpha} \int_{T_{1}}^{t}(t-q s)_{q}^{\alpha-1}[r(s)+H(s)] \nabla_{q} s
$$

for $t \geq T_{2}$. Thus, we get

$$
\limsup _{t \rightarrow \infty} t^{1-\alpha} \int_{T_{1}}^{t}(t-q s)_{q}^{\alpha-1}[r(s)+H(s)] \nabla_{q} s \leq\left|b_{1}\right|+c_{1}\left(T_{1}, T_{2}\right)+M_{2} \Gamma_{q}(\alpha)<\infty,
$$

which contradicts condition (30).

Case (2): Let $\alpha>1$. Then (24) and (25) are still true. Hence from (33) and using (32) we find that

$$
M_{2} \Gamma_{q}(\alpha) t^{1-\alpha} \geq-c_{2}\left(T_{2}\right)-c_{3}\left(T_{1}\right)+t^{1-\alpha} \int_{T_{1}}^{t}(t-q s)_{q}^{\alpha-1}[r(s)+H(s)] \nabla_{q} s
$$

for $t \geq T_{2}$. Since $\lim _{t \rightarrow \infty} t^{1-\alpha}=0$ for $\alpha>1$, we conclude

$$
\limsup _{t \rightarrow \infty} t^{1-\alpha} \int_{T_{1}}^{t}(t-q s)_{q}^{\alpha-1}[r(s)+H(s)] \nabla_{q} s \leq c_{2}\left(T_{2}\right)+c_{3}\left(T_{1}\right)<\infty,
$$

which contradicts condition (30). Therefore, we conclude that $x(t)$ is oscillatory. In case $x(t)$ is eventually bounded negative, similar arguments lead to a contradiction with condition (31).

\section{Oscillation of Caputo $q$-fractional difference equations}

In this section, we study the oscillation of the $q$-fractional difference equations of the form

$$
\left\{\begin{array}{l}
{ }_{q} C_{a}^{\alpha} x(t)+f_{1}(t, x)=r(t)+f_{2}(t, x), \quad t>a \geq 0, \\
\nabla_{q}^{k} x(a)=b_{k} \quad(k=0,1, \ldots, m-1),
\end{array}\right.
$$


where $m=\lceil\alpha\rceil$, and ${ }_{q} C_{a}^{\alpha}$ denotes the Liouville-Caputo (left) $q$-fractional derivative defined by (8). Notice that the Caputo differential operator ${ }_{q} C_{a}^{\alpha}$ demands functions to be $m$ times differentiable. Using (10), the solution representation of (34) is given by

$$
x(t)=\sum_{k=0}^{m-1} b_{k} \frac{(t-a)_{q}^{k}}{\Gamma_{q}(k+1)}+\frac{1}{\Gamma_{q}(\alpha)} \int_{a}^{t}(t-q s)_{q}^{\alpha-1} F(s, x) \nabla_{q} s
$$

for $t>a$ and $F(t, x)=r(t)+f_{2}(t, x)-f_{1}(t, x)$.

The oscillation of (34) is similar to the oscillation of (3). The only difference is the appearance of $t^{1-m}$ instead of $t^{1-\alpha}$ in the conditions. So we state the theorems without proofs.

Theorem 4.1 Let $f_{2}=0$ in (34) and condition (13) hold. If

$$
\liminf _{t \rightarrow \infty} t^{1-m} \int_{0}^{t}(t-q s)_{q}^{\alpha-1} r(s) \nabla_{q} s=-\infty
$$

and

$$
\limsup _{t \rightarrow \infty} t^{1-m} \int_{0}^{t}(t-q s)_{q}^{\alpha-1} r(s) \nabla_{q} s=\infty
$$

then every solution of (34) is oscillatory.

Theorem 4.2 Let conditions (13) and (14) hold with $\beta>\gamma$. If

$$
\liminf _{t \rightarrow \infty} t^{1-m} \int_{T}^{t}(t-q s)_{q}^{\alpha-1}[r(s)+H(s)] \nabla_{q} s=-\infty
$$

and

$$
\limsup _{t \rightarrow \infty} t^{1-m} \int_{T}^{t}(t-q s)_{q}^{\alpha-1}[r(s)-H(s)] \nabla_{q} s=\infty
$$

for every sufficiently large $T$, where $H$ is defined as in Theorem 3.3, then every solution of (34) is oscillatory.

Theorem 4.3 Let $\alpha \geq 1$ and suppose that (13) and (15) hold with $\beta<\gamma$.If

$$
\limsup _{t \rightarrow \infty} t^{1-m} \int_{T}^{t}(t-q s)_{q}^{\alpha-1}[r(s)+H(s)] \nabla_{q} s=\infty
$$

and

$$
\liminf _{t \rightarrow \infty} t^{1-m} \int_{T}^{t}(t-q s)_{q}^{\alpha-1}[r(s)-H(s)] \nabla_{q} s=-\infty
$$

for every sufficiently large $T$, where $H$ is defined as in Theorem 3.3, then every bounded solution of (34) is oscillatory. 


\section{Examples}

In this section, we construct numerical examples to illustrate the effectiveness of our theoretical results.

Example 5.1 Consider the Riemann-Liouville $q$-fractional difference equation

$$
\left\{\begin{array}{l}
{ }_{q} \nabla_{0}^{\alpha} x(t)+x^{5}(t) \ln (t+e)=\frac{(1+q) t^{2-\alpha}}{\Gamma_{q}(3-\alpha)}+\left(t^{10}-t^{\frac{2}{3}}\right) \ln (t+e)+x^{\frac{1}{3}}(t) \ln (t+e), \\
\lim _{t \rightarrow 0^{+}} I_{0}^{1-\alpha} x(t)=0, \quad 0<\alpha<1,
\end{array}\right.
$$

where $m=1, f_{1}(t, x)=x^{5}(t) \ln (t+e), r(t)=\frac{(1+q) t^{2-\alpha}}{\Gamma_{q}(3-\alpha)}+\left(t^{10}-t^{\frac{2}{3}}\right) \ln (t+e)$ and $f_{2}(t, x)=$ $x^{\frac{1}{3}}(t) \ln (t+e)$. It is easy to verify that conditions (13) and (14) are satisfied for $\beta=5, \gamma=\frac{1}{3}$ and $p_{1}(t)=p_{2}(t)=\ln (t+e)$. However, we show in the following that condition (26) does not hold. For every sufficiently large $T \geq 1$ and all $t \geq T$, we have $r(t)>0$. Calculating $H(s)$ as defined in Theorem 3.3, we find that $H(s) \simeq 0.77 \ln (s+e) \geq 0.77$. Then, using (5) and (11) with $\mu=0$, we get

$$
\begin{aligned}
\liminf _{t \rightarrow \infty} t^{1-\alpha} \int_{T}^{t}(t-q s)_{q}^{\alpha-1}[r(s)+H(s)] \nabla_{q} s & \geq \liminf _{t \rightarrow \infty} t^{1-\alpha} \int_{T}^{t}(t-q s)_{q}^{\alpha-1} H(s) \nabla_{q} s \\
& \geq \liminf _{t \rightarrow \infty} 0.77 t^{1-\alpha} \int_{T}^{t}(t-q s)_{q}^{\alpha-1} \cdot 1 \nabla_{q} s \\
& =\liminf _{t \rightarrow \infty} \frac{0.77 t^{1-\alpha}(t-T)_{q}^{\alpha}}{\Gamma_{q}(\alpha+1)} \\
& =\infty .
\end{aligned}
$$

However, one can easily verify that $x(t)=t^{2}$ is a nonoscillatory solution of (35) since

$$
{ }_{q} I_{0}^{1-\alpha}\left(t^{2}\right)=\frac{(1+q) t^{3-\alpha}}{\Gamma_{q}(4-\alpha)}
$$

and

$$
{ }_{q} \nabla_{0}^{\alpha}\left(t^{2}\right)=\frac{(1+q) t^{2-\alpha}}{\Gamma_{q}(3-\alpha)} .
$$

Example 5.2 Consider the Riemann-Liouville $q$-fractional difference equation

$$
\left\{\begin{array}{l}
{ }_{q} \nabla_{0}^{\alpha} x(t)+x^{3}(t)=\sin t, \\
\lim _{t \rightarrow 0^{+}} I_{0}^{1-\alpha} x(t)=0, \quad 0<\alpha<1,
\end{array}\right.
$$

where $f_{1}(t, x)=x^{3}(t), r(t)=\sin t$ and $f_{2}(t, x)=0$. Then condition (13) holds. Furthermore, one can easily check that

$$
\liminf _{t \rightarrow \infty} t^{1-\alpha} \int_{0}^{t}(t-q s)_{q}^{\alpha-1} \sin s \nabla_{q} s=-\infty
$$

and

$$
\limsup _{t \rightarrow \infty} t^{1-\alpha} \int_{0}^{t}(t-q s)_{q}^{\alpha-1} \sin s \nabla_{q} s=\infty .
$$


This shows that conditions (18) and (19) of Theorem 3.2 hold. Hence, every solution of (36) is oscillatory.

\section{Conclusion}

In this article, the oscillation theory for $q$-fractional difference equations was studied. Sufficient conditions for the oscillation of solutions of Riemann $q$-fractional difference equations of the form (3) were given in three theorems in Section 3. The work of Grace et al. [1] and Chen et al. [2] in fractional calculus was combined to study the oscillation theory for $q$-fractional difference equations of the form (3). The main approach is based on applying Young's inequality which will help us in obtaining sharper conditions. The oscillation for the Caputo $q$-fractional difference operators has been investigated as well. Numerical examples are presented to demonstrate the effectiveness of the obtained results.

Competing interests

The author declares that they have no competing interests.

Authors' contributions

All authors read and approved the final manuscript.

\section{Publisher's Note}

Springer Nature remains neutral with regard to jurisdictional claims in published maps and institutional affiliations.

Received: 8 May 2017 Accepted: 9 August 2017 Published online: 24 August 2017

References

1. Grace, SR, Agarwal, RP, Wong, PJY, Zafer, A: On the oscillation of fractional differential equations. Fract. Calc. Appl. Anal. 15, 222-231 (2012). doi:10.2478/s13540-012-0016-1

2. Chen, D-X, Qu, P-X, Lan, Y-H: Forced oscillation of certain fractional differential equations. Adv. Differ. Equ. 2013(1), Article ID 125 (2013). doi:10.1186/1687-1847-2013-125

3. Alzabut, J, Abdeljawad, T: Sufficient conditions for the oscillation of nonlinear fractional difference equations. J. Fract. Calc. Appl. 5(1), 177-187 (2014)

4. Abdalla, B, Abodayeh, K, Abdeljawad, T, Alzabut, J: New oscillation criteria for forced nonlinear fractional difference equations. Vietnam J. Math. (2016). doi:10.1007/s10013-016-0230-y

5. Annaby, MH, Mansour, ZS: q-Fractional Calculus and Equations. Springer, Berlin (2012)

6. Abdeljawad, T, Baleanu, D: Caputo $q$-fractional initial value problems and a $q$-analogue Mittag-Leffler function. Commun. Nonlinear Sci. Numer. Simul. 16(12), 4682-4688 (2011)

7. Jarad, F, Abdeljawad, T, Gundogdu, E, Baleanu, D: On the Mittag-Leffler stability of $q$-fractional nonlinear dynamical systems. Proc. Rom. Acad., Ser. A: Math. Phys. Tech. Sci. Inf. Sci. 12(4), 309-314 (2011)

8. Abdeljawad, T, Alzabut, J: The $q$-fractional analogue for Gronwall-type inequality. J. Funct. Spaces Appl. 2013, Article ID 543839 (2013)

9. Abdeljawad, T, Benli, B, Baleanu, D: A generalized q-Mittag-Leffler function by $q$-Caputo fractional linear equations. Abstr. Appl. Anal. 2012, Article ID 546062 (2012)

10. Wu, G-C, Baleanu, D: New applications of the variational iteration method - from differential equations to $q$-fractional difference equations. Adv. Differ. Equ. 2013(1), Article ID 21 (2013). doi:10.1186/1687-1847-2013-21

11. Abdeljawad, T, Alzabut, J, Baleanu, D: A generalized $q$-fractional Gronwall inequality and its applications to nonlinear delay $q$-fractional difference systems. J. Inequal. Appl. 2016(1), Article ID 240 (2016). doi:10.1186/s13660-016-1181-2

12. Coronel-Escamilla, A, Gómez-Aguilar, JF, Baleanu, D, Córdova-Fraga, T, Escobar-Jiménez, RF, Olivares-Peregrino, VH, Qurashi, MMA: Bateman-Feshbach Tikochinsky and Caldirola-Kanai oscillators with new fractional differentiation. Entropy 19(2), Article ID 55 (2017)

13. Atıcı, FM, Eloe, PW: Fractional q-calculus on a time scale. J. Nonlinear Math. Phys. 14(3), 333-344 (2007)

14. Hardy, GH, Littlewood, JE, Polya, G: Inequalities, 2nd edn. Cambridge University Press, Cambridge (1988) 